\title{
Competence versus mastery: The time course for developing proficiency in video-assisted thoracoscopic lobectomy
}

\author{
Xiao Li, MD, ${ }^{a}$ Jun Wang, MD, ${ }^{a}$ and Mark K. Ferguson, MD $^{\mathrm{b}}$
}

\begin{abstract}
Objectives: Thoracoscopic lobectomy has a vaguely defined learning curve for competency, whereas the development of proficiency has not been evaluated. We compared learning curves for 2 surgeons experienced in open lobectomy to define the learning process for thoracoscopic lobectomy.
\end{abstract}

\begin{abstract}
Methods: The first 200 patients who underwent thoracoscopic lobectomy by 1 senior surgeon at 2 different institutions were evaluated. Data were abstracted from prospectively maintained databases. Learning curves were evaluated for operative time, blood loss, and postoperative length of stay by assessing elements of proficiency: efficiency (defined as decreasing values for these variables, assessed by Change-Point Analysis) and consistency (defined as the absence of outliers, evaluated by moving average). Conversion to open rates and complication rates were assessed.
\end{abstract}

Results: Surgeon A's patients were younger than Surgeon B's patients $(57.4$ vs 66.0 years; $P<.001)$ and had fewer medical comorbidities. For Surgeons A and B, operation time (mean, 178 vs 180 minutes) efficiency was achieved at 157 and 108 cases, respectively, and blood loss (mean 181 vs $178 \mathrm{~mL}$ ) efficiency was achieved at 126 and 139 cases, respectively. Conversion to open rates decreased between the first and second halves of the study $(P<.001)$ despite expanding anatomic indications for a video-assisted thoracic surgery approach. Consistency was not reliably achieved for either surgeon for operating time or blood loss. Postoperative length of stay and complication rates did not change for either surgeon.

Conclusions: The learning curves for video-assisted thoracoscopic lobectomy were similar for both surgeons. Between 100 and 200 cases are required to achieve efficiency, and consistency requires even more cases. (J Thorac Cardiovasc Surg 2014;147:1150-4)

Competence is the benchmark by which physicians are permitted to perform procedures independently. Developing competence in a complex operation entails performing a sufficient number of procedures to demonstrate consistent safety and efficacy. For minimally invasive lobectomy, this appears to require a minimum of 20 to 30 cases, with estimates as high as 50 operations. ${ }^{1-4}$ Progressing to proficiency in a complex procedure not only necessitates substantial additional operative experience but also requires a qualitative leap in knowledge and performance. ${ }^{5}$ Two measures that characterize proficiency are efficiency and consistency. The learning curves that describe the achievement of proficiency are likely to be different than those for developing competency.

From the Department of Thoracic Surgery, ${ }^{a}$ Peking University People's Hospital, Beijing, China; and Department of Surgery and Cancer Research Center, ${ }^{\mathrm{b}}$ The University of Chicago, Chicago, Ill.

Disclosures: Authors have nothing to disclose with regard to commercial support.

Read at the 39th Annual Meeting of The Western Thoracic Surgical Association, Coeur d'Alene, Idaho, June 26-29, 2013.

Received for publication June 18, 2013; revisions received Oct 21, 2013; accepted for publication Nov 26, 2013; available ahead of print Jan 10, 2014.

Address for reprints: Mark K. Ferguson, MD, Section of Cardiac and Thoracic Surgery, The University of Chicago, 5841 S. Maryland Ave MC5040, Chicago, IL 60637 (E-mail: mferguso@ surgery.bsd.uchicago.edu).

$0022-5223 / \$ 36.00$

Copyright (C) 2014 by The American Association for Thoracic Surgery

http://dx.doi.org/10.1016/j.jtcvs.2013.11.036
Determining the time and case number required to attain procedural proficiency is important for many reasons. When feasible, newly trained surgeons should be mentored until they demonstrate skills and outcomes that exceed those required for competency, and the time and manpower needed for such supervision need to be appropriately allocated. It is possible that medical-legal risk exposure and associated malpractice payments ultimately might be assessed on the basis of level of expertise, and knowledge of the duration of increased risk would help correctly apportion individual contributions. Understanding the time and caseload required to develop proficiency permits assessment of whether simulation or intense mentorship is able to shorten the duration of the learning curve.

We studied learning curves for video-assisted thoracic surgery (VATS) lobectomy to determine how long it takes to achieve proficiency by analyzing both intraoperative and postoperative factors for efficiency and consistency. We also assessed whether developing efficiency and consistency require similar time frames.

\section{MATERIALS AND METHODS}

The first 200 patients who underwent planned VATS lung resection by each of 2 senior surgeons at different institutions were evaluated. Major lung resections performed using an open thoracotomy also were catalogued for the same time period. Both surgeons had extensive prior experience with VATS before they began doing VATS lung resections and had 

Abbreviations and Acronyms
CUSUM $=$ cumulative sum
EBL $=$ estimated blood loss
VATS $=$ video-assisted thoracic surgery

extensive prior experience with open lung resection. Patients were included in the VATS analysis who had surgery for benign or malignant lung disease. Patients were excluded who underwent concomitant chest wall resection via VATS or robotic-assisted VATS. VATS lung resection was defined as lobectomy. VATS lung resection was performed using a 3-incision approach, including 2 ports and an access incision up to $5 \mathrm{~cm}$ in length, without rib spreading, with or without lymph node dissection as appropriate for the underlying disease.

Data were abstracted from prospectively maintained and internal review board-approved databases. Patient consent for this study was waived. Patient demographics and preoperative clinical factors were identified. Intraoperative data and details regarding the pathology specimen were collected. Conversion to an open procedure was defined as emergency or elective thoracotomy, regardless of length and that included rib spreading, during the VATS procedure. Pathologic staging, ${ }^{6}$ postoperative length of stay, and postoperative complications were analyzed. Operating time was defined as the time from incision to the time of skin closure. Estimated blood loss (EBL) was based on estimates by the surgeon and anesthesiologist. Postoperative complication data were collected from the hospital record prospectively and categorized as pulmonary (pneumonia, prolonged postoperative intubation, reintubation, lung collapse requiring bronchoscopy, acute respiratory distress syndrome), cardiovascular (pulmonary embolism, myocardial infarction, arrhythmia requiring intervention), and other. Operative mortality was defined as death occurring during hospitalization for lung surgery or within 30 days of the operation.

Statistical analyses were performed using Statistical Product and Service Solutions version 13.0 (SPSS Inc, Chicago, Ill). Continuous variables are reported as mean and standard deviation, and categoric variables are reported as frequency and proportion. Chi-square analysis or the Fisher exact test was used to assess differences in categoric data, and the $t$ test or Wilcoxon rank test was used to assess the differences between continuous variables. The changes in operative times, EBL, and length of stay were assessed using Change-Point Analysis (Taylor Enterprises, Inc, Libertyville, Ill) with bootstrapping (1000 bootstraps) for evaluation of efficiency. Change-Point Analysis is a variant of cumulative sum (CUSUM) analysis that was developed to detect significant changes in time series data. For interpretation of graphic representations, values on the upward slope of a Change-Point curve tend to be greater than average, and values on the downward slope of a curve tend to be less than average. Analysis was also performed with the moving average technique to assess consistency by identifying outliers, using means and standard deviations of data for the last 50 patients of each surgeon as the limits to which the data were compared. The sample size and moving average length were both set at 10 , resulting in 20 sampled intervals for the 200 patients, and outliers were identified that were greater than 1 standard deviation from the mean.

\section{RESULTS}

A total of 400 patients were evaluated for this study. Surgeon A began performing VATS lobectomy in September 2006 and completed 200 attempted cases by May 2010, an interval of 43.2 months. Surgeon B began performing VATS lobectomy in March 2007 and completed 200 attempted cases by October 2012, an interval of 68.6 months. Patient characteristics are listed in Table 1. The patient populations differed in a number of characteristics, including age, gender, and medical comorbidities. There were no differences between the groups in performance status or baseline lung function.

Surgical outcomes are listed in Table 2. There were similarities in types of operations, operative times, and surgical blood loss. Resection was performed more often for cancer by Surgeon B, and those operations were more often performed for earlier-stage disease. Clinical tumor size was similar for both surgeons. Length of stay was longer for Surgeon A. Conversion and incidence of complications were more common for Surgeon B. Surgeon A's conversion rates were $7 \%$ and $1 \%$ in the first and second halves of the study, respectively $(P=.03)$, and Surgeon B's conversion rates were $19 \%$ and $4 \%$, respectively $(P=.001)$. Complication rates for Surgeon A $(7 \%$ and $11 \%, P=.32)$ and Surgeon B $(28 \%$ and $20 \%, P=.19)$ were similar for both periods.

We grouped VATS and open cases by VATS 50-case intervals to examine whether surgeon selection of cases changed over the period of study (Table 3). The percentage of VATS cases pathologically staged greater than I or II increased significantly during the period of study. Tumor diameter trended upward during the period of study, but the differences did not reach statistical significance. Open cases performed during the study period (158) were classified according to indication as reoperation (21), induction chemoradiotherapy (26), robotic resection (4), and anatomy (central location, tumor size, extent of nodal involvement, suspected mediastinal or chest wall involvement, pneumonectomy; 107). The percent of cases performed open because of anatomic causes significantly decreased during the study period.

Change-Point analysis demonstrated that the inflection point for operating time for Surgeon A occurred at patient 157 (99\% confidence; case numbers 128-173), and the primary inflection point for Surgeon B occurred at patient 108 (100\% confidence; case numbers 90-120; Figure 1). For EBL, the inflection point for Surgeon A occurred at patient 126 (100\% confidence; case numbers 96-148), whereas for Surgeon B the primary inflection point occurred at patient 139 (100\% confidence; case numbers 132-153).

We grouped cases by 2 -month time intervals to investigate whether the time interval or case number is more important in reaching a Change-Point. The inflection point for operating time for Surgeon A occurred at time interval 19 (99\% confidence; time intervals 17-20), at which point Surgeon A had performed approximately 160 cases. In comparison, the primary inflection point for operating time for Surgeon B occurred at time interval $20(100 \%$ confidence; time intervals 17-21), at which time Surgeon B had performed approximately 110 cases. Inflection points for EBL were at time interval 17 ( $\sim 130$ cases) for Surgeon 
TABLE 1. Characteristics of patients undergoing attempted videoassisted thoracoscopic lobectomy

\begin{tabular}{lcccc}
\hline \multicolumn{1}{c}{ Variable } & Surgeon A & Surgeon B & $\boldsymbol{P}$ value & Overall \\
\hline Age $(\mathrm{y})$ & $57.3 \pm 13.8$ & $65.9 \pm 11.0$ & $<.001$ & $61.6 \pm 13.2$ \\
Male sex & $99(49.5)$ & $77(38.5)$ & .027 & $176(44.0)$ \\
Obese & $10(5.0)$ & $55(27.5)$ & $<.001$ & $65(16.3)$ \\
$\quad\left(\mathrm{BMI}>30 \mathrm{~kg} / \mathrm{m}^{2}\right)$ & & & & \\
Underweight & $7(3.5)$ & $5(2.5)$ & .56 & $12(3.0)$ \\
$\quad\left(\mathrm{BMI}<18.5 \mathrm{~kg} / \mathrm{m}^{2}\right)$ & & & & \\
Ever smoker & $64(32.0)$ & $161(80.5)$ & $<.001$ & $225(56.3)$ \\
Diabetes mellitus & $20(10.0)$ & $7(18.5)$ & .015 & $57(14.3)$ \\
Serum creatinine & $0.85 \pm 0.20$ & $0.97 \pm 0.65$ & .016 & $0.91 \pm 0.48$ \\
$\quad(\mathrm{mg} / \mathrm{dL})$ & & & & \\
Hypertension & $50(25.0)$ & $102(51.0)$ & $<.001$ & $152(38.0)$ \\
Coronary artery disease & $16(8.0)$ & $35(17.5)$ & .004 & $51(15.8)$ \\
PS 0-1 & $198(99.0)$ & $195(97.5)$ & .25 & $393(98.3)$ \\
FEV1\% & $93.1 \pm 16.8$ & $91.0 \pm 23.4$ & .32 & $92.0 \pm 20.6$ \\
DLCO\% & $84.1 \pm 14.9$ & $86.3 \pm 22.7$ & .25 & $85.3 \pm 19.4$ \\
\hline Co &
\end{tabular}

Continuous data are expressed as mean \pm standard deviation. Values in parentheses represent percentages for categoric data. $B M I$, Body mass index; $D L C O \%$, diffusing capacity for carbon monoxide expressed as a percent predicted; $F E V I \%$, forced expiratory volume during the first second expressed as a percent predicted; $P S$, performance status.

A and at time interval 24 ( $\sim 140$ cases) for Surgeon B. Hospital length of stay did not change during the study period for either surgeon.

Moving average analyses demonstrated that consistency for operating time occurred by the 18th sampled case interval for both surgeons (Figure 2). EBL did not reach consistency for Surgeon A by the end of the sample period and demonstrated consistency for surgeon B by the 18th sampled case interval. Hospital length of stay, in contrast,

TABLE 2. Operative and outcomes data for patients undergoing attempted video-assisted thoracoscopic lobectomy

\begin{tabular}{lcccc}
\hline \multicolumn{1}{c}{ Variable } & Surgeon A & Surgeon B & value & Overall \\
\hline Right side resection & $115(57.5)$ & $99(49.5)$ & .11 & $214(53.5)$ \\
Upper lobe resection & $96(48.0)$ & $12(56.0)$ & .11 & $208(52)$ \\
Conversion to open & $8(4.0)$ & $24(12.0)$ & .003 & $32(8.0)$ \\
Operation time & $178.7 \pm 52.4$ & $180.1 \pm 49.1$ & .79 & $179.4 \pm 50.7$ \\
EBL (mL) & $181.0 \pm 145.0178 .0 \pm 358.0$ & .91 & $179.4 \pm 272.9$ \\
Pulmonary & $10(5.0)$ & $21(10.5)$ & .04 & $31(7.8)$ \\
$\quad$ complications & & & & \\
Cardiovascular & $6(3.0)$ & $25(12.5)$ & $<.001$ & $31(7.8)$ \\
$\quad$ complications & & & & \\
Any complications & $18(9.0)$ & $48(24.0)$ & $<.001$ & $66(16.5)$ \\
Mortality & $1(0.5)$ & $4(2.0)$ & .18 & $5(1.3)$ \\
Length of stay (d) & $9.8 \pm 3.9$ & $4.5 \pm 3.4$ & $<.001$ & $7.2 \pm 4.5$ \\
Resection for cancer & $134(67.0)$ & $175(87.5)$ & $<.001$ & $309(77.3)$ \\
Tumor diameter & $27.1 \pm 19.4$ & $25.0 \pm 13.6$ & .25 & $26.0 \pm 16.7$ \\
$\quad$ (radiographic; mm) & & & & \\
Tumor stage I or II & $108(77.1)$ & $168(94.4)$ & $<.001$ & $276(86.8)$ \\
\hline
\end{tabular}

Continuous data are expressed as mean \pm standard deviation. Values in parentheses represent percentages for categoric data. $E B L$, Estimated blood loss. was within the 1 standard deviation limit for both surgeons for almost the entire observation period.

\section{DISCUSSION}

The degree of procedural expertise exhibited by a trainee or practicing physician is often difficult to categorize. In the United States, certifying organizations typically require that physicians demonstrate "competence" in a specialty area to qualify to practice in that area. From a procedural perspective, "competent" indicates that the physician has the ability to perform a procedure safely and effectively. This is a minimum standard; certainly most patients hope that their physicians possess greater expertise in procedural skills. Greater expertise indicates that the physician has gained additional experience, learned how to avoid common errors, and developed resiliency in the face of unexpected challenges or events during the perioperative period. This leads to integrated understanding of clinical and technical challenges and improved situational awareness, which permit both analytic and intuitive decision-making. When compared with a "competent" performer, one who is "proficient" will demonstrate, in addition to safety and efficacy, both efficiency and consistency.

In this study evaluating the development of proficiency in VATS lobectomy, CUSUM analysis was used to evaluate efficiency. Efficiency was defined as refining performance to decrease operating time, EBL, and hospital stay. We found that efficiency was achieved at different points for operating time and EBL, and no efficiency was achieved for length of stay. In evaluating whether case number or the relative frequency of cases was the dominant factor in achieving efficiency, we obtained mixed results. Both surgeons achieved efficiency in operating time at almost the same time interval, which represented 30\% fewer cases for Surgeon B than for Surgeon A. This suggested that time interval rather than case frequency or case volume was more important. In contrast, efficiency in EBL was achieved by the 2 surgeons 14 weeks apart, but required similar case numbers for both surgeons. This suggests that case number is more important than time interval for attaining efficiency in EBL. It is possible that different learning curves and determinants of efficiency are applicable to these different end points.

Another measure of proficiency in performing an operation is consistency, which was evaluated using the moving average method. For our study, consistency was defined as eliminating outliers in terms of operating time, EBL, hospital length of stay, and conversion rates. Consistency in operating time seemed to develop just at the end of the study period. In contrast, consistency was not reliably achieved for EBL during the study period. This should not be surprising, because VATS is associated with an increased risk of complications compared with 
TABLE 3. Evolution of indications for video-assisted thoracoscopic lobectomy over time

\begin{tabular}{|c|c|c|c|c|c|}
\hline Variable & First 50 cases & Second 50 cases & Third 50 cases & Fourth 50 cases & $P$ value \\
\hline Stages III-IV & 7 & 6 & 14 & 15 & .047 \\
\hline Tumor diameter on CT (mm) & $23.6 \pm 11.9$ & $24.5 \pm 13.4$ & $27.7 \pm 21.1$ & $28.2 \pm 18.5$ & .186 \\
\hline Pathologic tumor diameter (mm) & $23.3 \pm 12.3$ & $23.9 \pm 13.9$ & $24.4 \pm 17.7$ & $26.1 \pm 18.3$ & .705 \\
\hline Open resections because of anatomy & 50 & 31 & 15 & 11 & $<.001$ \\
\hline
\end{tabular}

Case categories are listed for each surgeon; each column represents 100 total cases. Continuous data are expressed as mean \pm standard deviation. $C T$, Computed tomography.

open operations ${ }^{7}$ that typically are related to bleeding ${ }^{8}$ and have not been etiologically related to surgeon experience. Hospital length of stay did not change appreciably during the study period, and so consistency was achieved for that measure.

The number of conversions required to successfully complete a lobectomy is one measure of surgical quality. Conversion rates were significantly reduced for both surgeons during the second half of the study, indicating an improved ability to avoid and manage problems, which is a sign of resiliency. This change occurred over a time period during which VATS case selection criteria evolved as evidenced by increasing tumor diameter and cancer stage, and by an increasing willingness to perform VATS resections in patients with anatomically more challenging tumors. Complication rates did not change during the study period, indicating that overall quality remained stable during a time when efficiency and possibly consistency were achieved.

The time frame for achieving proficiency in surgery has not been extensively studied. One report that evaluated proficiency in hand-assisted laparoscopic colon and rectal

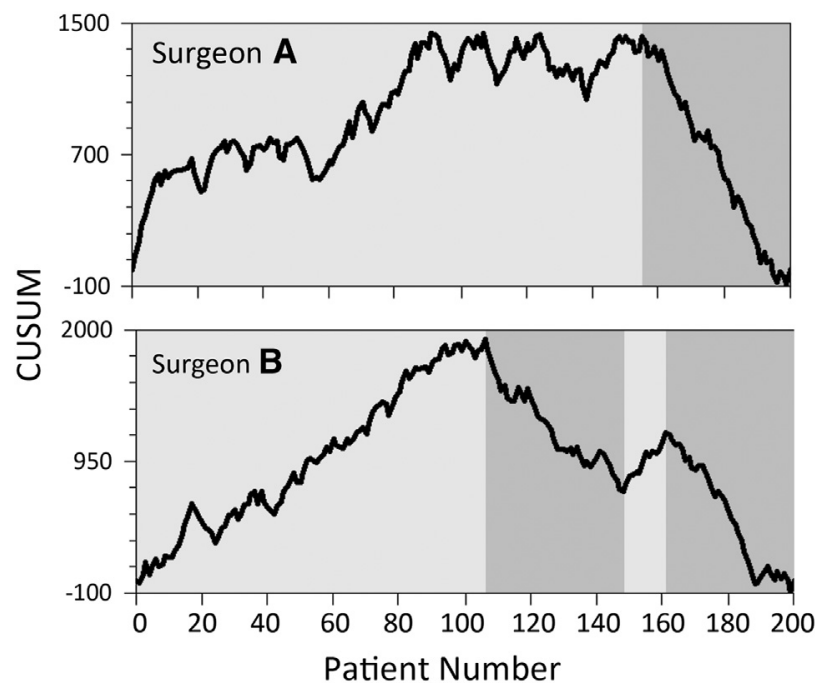

FIGURE 1. Change-Point Analysis of operating time for Surgeons A (top) and B (bottom). An upward slope (lighter background) indicates that values were generally greater than the mean for the group, and a downward slope (darker background) indicates that values were generally less than the mean for the group. CUSUM, Cumulative sum. surgery identified a change-point in operative time at 105 and 108 cases for each of 2 surgeons, which is similar to our findings for VATS lobectomy. ${ }^{9}$ CUSUM analysis has been used to investigate the learning curve for outcomes for paraesophageal hiatal hernia repair, demonstrating that the inflection point for recurrence occurred at case $18 .^{10}$ Although some of the statistical techniques used in evaluating hernia repair were similar to those in the present study, the outcome in the hernia study was a measure of efficacy/competence rather than proficiency, and the results compare closely with other estimates of case numbers needed to achieve competence. Other evaluations indicate that there is a continued decrease in operating time as

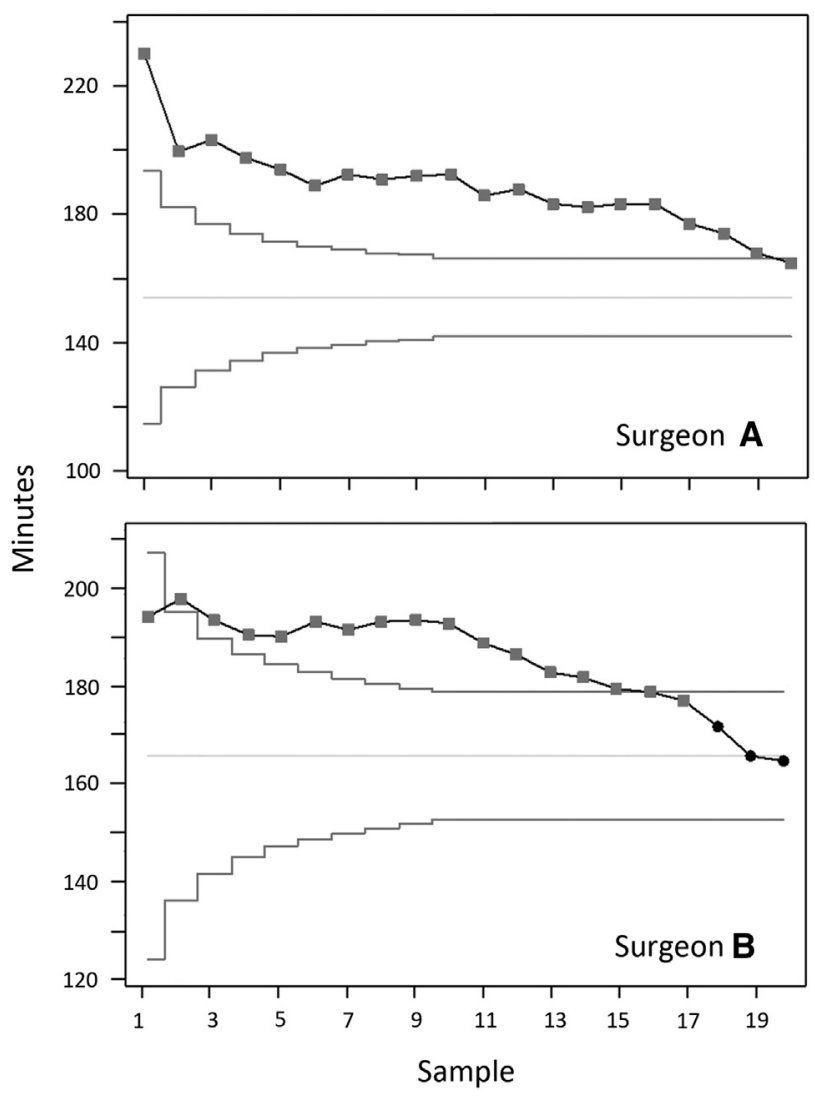

FIGURE 2. Moving average illustration of operating time for Surgeons A (top) and B (bottom). Values represented by squares indicate that at least 1 measurement during the period was greater than 1 standard deviation from the mean. Mean values were calculated from the last 50 cases in each series. 
surgeon experience increases, even after hundreds of operations. ${ }^{11,12}$ However, these studies did not determine when efficiency is first achieved.

Examination of the learning curve for proficiency should have no direct bearing on whether surgeons are competent to perform complex operations independently. Regulatory organizations and individual hospitals set the necessary standards and render decisions about an individual surgeon's qualifications. The importance of assessing the time course for developing proficiency lies in identifying resource expenditures that professional mentorship requires, the potential need for institutional oversight because of possible safety concerns, and the evaluation of the role of simulation training in shortening the learning curve. It is often the custom in group practices to have a senior surgeon monitor the performance of a newly finished trainee who has joined the group to ensure that their new colleague has a safe initial experience. Few partners would predict that such monitoring may need to last for years rather than months. The surgeons involved in this study had no VATS mentorship available as their level of expertise developed; the presence of mentorship may substantially shorten the time and case load required to achieve proficiency.

Believing that physicians just out of training or who are beginning to perform new procedures will likely be merely competent and have a higher risk of complications and other adverse outcomes, ${ }^{13}$ malpractice insurers may want to know for how long such additional risk exposure exists. Simulation may be able to replace some of the case numbers required to achieve proficiency in a complex operation, but this requires that the simulation environment have high fidelity and be challenging, constantly testing the learner's growing cognitive and technical skills. ${ }^{14}$ Such training for VATS lobectomy has yet to be developed.

\section{Study Limitations}

There are a number of potential drawbacks to this study. The use of data from a small number of surgeons limits the application of our findings to a general population of learners, but this approach has been the standard for assessing learning curves in a series of publications, including a number of recent reports. ${ }^{9-11,15,16}$ The use of data from different continents and cultures created potentially important differences between the patient groups, including preoperative risk factors and perioperative management algorithms. How these might have affected the study results is unknown. Finally, there is no clear definition of proficiency that can be translated into clinical research terms. For this study, we focused on efficiency and consistency, but other factors such as quality improvement and cost containment may be reasonable study end points.

\section{CONCLUSIONS}

Achieving proficiency in performing VATS lobectomy seems to require more than 100 cases of personal experience to develop efficiency, and attaining consistency requires 200 or more cases. Further evaluation of the time course required to achieve proficiency could provide insight into the underlying costs of a surgeon's post-training clinical development and might suggest methods that could help shorten the learning curve.

\section{References}

1. Zhao H, Bu L, Yang F, Li J, Li Y, Wang J. Video-assisted thoracoscopic surgery lobectomy for lung cancer: the learning curve. World J Surg. 2010;34:2368-72

2. Konge L, Petersen RH, Hansen HJ, Ringsted C. No extensive experience in open procedures is needed to learn lobectomy by video-assisted thoracic surgery. Interact Cardiovasc Thorac Surg. 2012;15:961-5.

3. Meyer M, Gharagozloo F, Tempesta B, Margolis M, Strother E, Christenson D. The learning curve of robotic lobectomy. Int J Med Robot. 2012;8:448-52.

4. Ra YJ, Ahn HY, Kim MS. Learning curve of a young surgeon's video-assisted thoracic surgery lobectomy during his first year experience in newly established institution. Korean J Thorac Cardiovasc Surg. 2012;45:166-70.

5. Dreyfus SE, Dreyfus HL. A five-stage model of the mental activities involved in directed skill acquisition. Operations Research Center, ORC 80-2, University of California Berkeley, Feb 1980.

6. American Joint Committee on Cancer. AJCC Cancer Staging Manual. 7th ed. New York, NY: Springer; 2010.

7. Gopaldas RR, Bakaeen FG, Dao TK, Walsh GL, Swisher SG, Chu D. Video-assisted thoracoscopic versus open thoracotomy lobectomy in a cohort of 13,619 patients. Ann Thorac Surg. 2010;89:1563-70.

8. Flores RM, Thekweazu U, Dycoco J, Rizk NP, Rusch VW, Bains MS, et al. Video-assisted thoracoscopic surgery (VATS) lobectomy: catastrophic intraoperative complications. J Thorac Cardiovasc Surg. 2011;142:1412-7.

9. Pendlimari R, Holubar SD, Dozois EJ, Larson DW, Pemberton JH, Cima RR. Technical proficiency in hand-assisted laparoscopic colon and rectal surgery determining how many cases are required to achieve mastery. Arch Surg. 2012; 147:317-22.

10. Okrainec A, Ferri LE, Feldman LS, Fried GM. Defining the learning curve in laparoscopic paraesophageal hernia repair: a CUSUM analysis. Surg Endosc. 2011;25:1083-7.

11. Ballantyne GH, Ewing D, Capella RF, Capella JF, Davis D, Schmidt HJ, et al. The learning curve measured by operating times for laparoscopic and open gastric bypass: roles of surgeon's experience, institutional experience, body mass index and fellowship training. Obes Surg. 2005;15:172-82.

12. Tekkis PP, Senagore AJ, Delaney CP, Fazio VW. Evaluation of the learning curve in laparoscopic colorectal surgery: comparison of right-sided and left-sided resections. Ann Surg. 2005;242:83-91.

13. David G, Gunnarsson CL, Moore M, Howington J, Miller DL, Maddaus MA, et al. Surgeons' volume-outcome relationship for lobectomies and wedge resections for cancer using video-assisted thoracoscopic techniques. Minim Invasive Surg. 2012;2012:760292.

14. Cristancho SM, Moussa F, Dubrowski A. A framework-based approach to designing simulation-augmented surgical education and training programs. Am J Surg. 2011;202:344-51.

15. Ramsay CR, Grant AM, Wallace SA, Garthwaite PH, Monk AF, Russell IT. Statistical assessment of the learning curves of health technologies. Health Technol Assess. 2001;5:1-98.

16. Charland PJ, Robbins T, Rodriguez E, Nifong WL, Chitwood RW Jr. Learning curve analysis of mitral valve repair using telemanipulative technology. J Thorac Cardiovasc Surg. 2011;142:404-10. 